La distribution des électrons

dans un cristal peut être

reconstruite avec une grande

précision au moyen

de la diffraction des rayons $X$

à haute résolution, alors que

la diffraction des neutrons

polarisés en spin permet

de retrouver la densité

d'aimantation. Bien que

les grandeurs ainsi mesurées

soient toutes deux une

traduction du comportement

des électrons, ces deux types

d'expérience de diffusion

sont de nos jours interprétées

par des modèles différents.

Nous retraçons ici les étapes

ayant conduit à la première

détermination expérimentale

de la densité d'électrons

résolue en spin,

par un traitement combiné

des données de diffraction

de rayons $X$ et de neutrons

polarisés.

\title{
Les rayons $X$ et les neutrons se combinent pour révéler la densité électronique résolue en spin
}

Maxime Deutsch ${ }^{(1,2,3)}$, Nicolas Claiser ${ }^{(2)}$ (nicolas.laiser@univ-lorraine.fr),

Béatrice Gillon ${ }^{(1)}$ (beatrice.gillon@(ea.fr), Claude Lecomte ${ }^{(2)}$, Mohamed Souhassou ${ }^{(2)}$,

Dominique Luneau $^{(4)}$ et Jean-Michel Gillet ${ }^{(5)}$ (jean-michel.gillet@ecp.fr)

(1) Laboratoire Léon Brillouin, CEA-CNRS (UMR 12), CEA/Saclay, 91191 Gif-sur-Yvette Cedex

(2) CRM2, CNRS-Université de Lorraine, BP 70239, 54506 Vandouvre-lès-Nancy Cedex

(3) Synchrotron S0LElL, L'Orme des Merisiers, St Aubin, 91192 Gif-sur-Yvette

(4) LMI (UMR 5615), Université Claude Bernard Lyon 1, 69622 Villeurbanne Cedex

(5) Laboratoire SPMS (UMR 8580), Université Paris-Saclay, CentraleSupelec, CNRS, Grande Voie des Vignes,

92295 Chatenay-Malabry Cedex

Toute la subtilité des interactions chimiques, la force des liaisons, la cohésion des molécules et cristaux se retrouvent dans le faible écart entre distribution réelle des électrons dans le cristal et une répartition obtenue par simple addition des densités électroniques d'atomes sans interactions. Cette grandeur, nommée "densité électronique de déformation ", représente les migrations de charges électroniques qui ont conduit à l'établissement des liaisons chimiques. Elle peut être obtenue par la diffraction des rayons $\mathrm{X}$ à haute résolution.

Lorsqu'on s'intéresse en plus aux propriétés magnétiques, il faut non seulement connaître la répartition spatiale des électrons mais aussi leur état de spin qui conditionne l'orientation du moment magnétique associé. Or, suite au principe de Pauli, les contributions magnétiques des électrons appariés dans une même orbitale s'annulent deux à deux et, sur une grande assemblée d'électrons dans une molécule ou une maille cristalline, seuls des électrons " isolés ", dits " non appariés ", vont pouvoir apporter une contribution utile aux propriétés magnétiques. Ce sont donc ces électrons particuliers qui doivent être étudiés préférentiellement pour comprendre les mécanismes à l'origine du magnétisme de spin. Pour avoir accès à ces propriétés, on utilise la diffraction de neutrons polarisés qui permet de reconstruire la densité de spin : $s(\mathbf{r})=\rho_{\uparrow}(\mathbf{r})-\rho_{\downarrow}(\mathbf{r})$, sachant que la diffraction des rayons $\mathrm{X}$ donne accès à la densité électronique : $\rho(\mathbf{r})=\rho_{\uparrow}(\mathbf{r})+\rho_{\downarrow}(\mathbf{r})$, où $\rho_{\uparrow}(\mathbf{r})$ et $\rho_{\downarrow}(\mathbf{r})$ sont les densités de présence respectives des électrons de spin $\uparrow$ (dit majoritaire) et de spin $\downarrow$ (dit minoritaire).
Le but de cet article est de montrer que la détermination expérimentale de la densité électronique des électrons a atteint un point où il est devenu possible de modéliser et de séparer leurs contributions en fonction de leur état de spin, grâce à l'utilisation conjointe d'expériences de diffraction $\mathrm{X}$ et de neutrons. À titre d'illustration, nous présentons la première reconstruction expérimentale d'une distribution d'électrons résolue en spin $\left(\rho_{\uparrow}(\mathbf{r})\right.$ et $\left.\rho_{\downarrow}(\mathbf{r})\right)$ dans un cristal moléculaire magnétique.

\section{Les rayons $X$, la charge et le modèle de pseudo-atomes}

La diffraction des rayons X (DRX) donne un accès - partiel - aux coefficients de Fourier de la distribution des électrons dans le cristal. Ces coefficients sont appelés "facteurs de structure " et notés $F_{\mathrm{e}} \operatorname{DRX}_{(\mathrm{H})}$, où les vecteurs $\mathrm{H}$, de coordonnées entières, décrivent le réseau réciproque du cristal. La distribution des électrons est ensuite reconstruite à l'aide d'un modèle, le plus populaire étant celui des "pseudo-atomes déformés » proposé par Hansen et Coppens en 1978 [1]. Ce modèle exprime la densité électronique du cristal comme une somme de densités centrées sur chaque noyau (" pseudo-atomes »), qui tient compte des transferts de charges et des déformations de la densité électronique de valence.

Pour chacun de ces pseudo-atomes, il convient donc de proposer une forme analytique de la densité électronique qui possède suffisamment de degrés de 

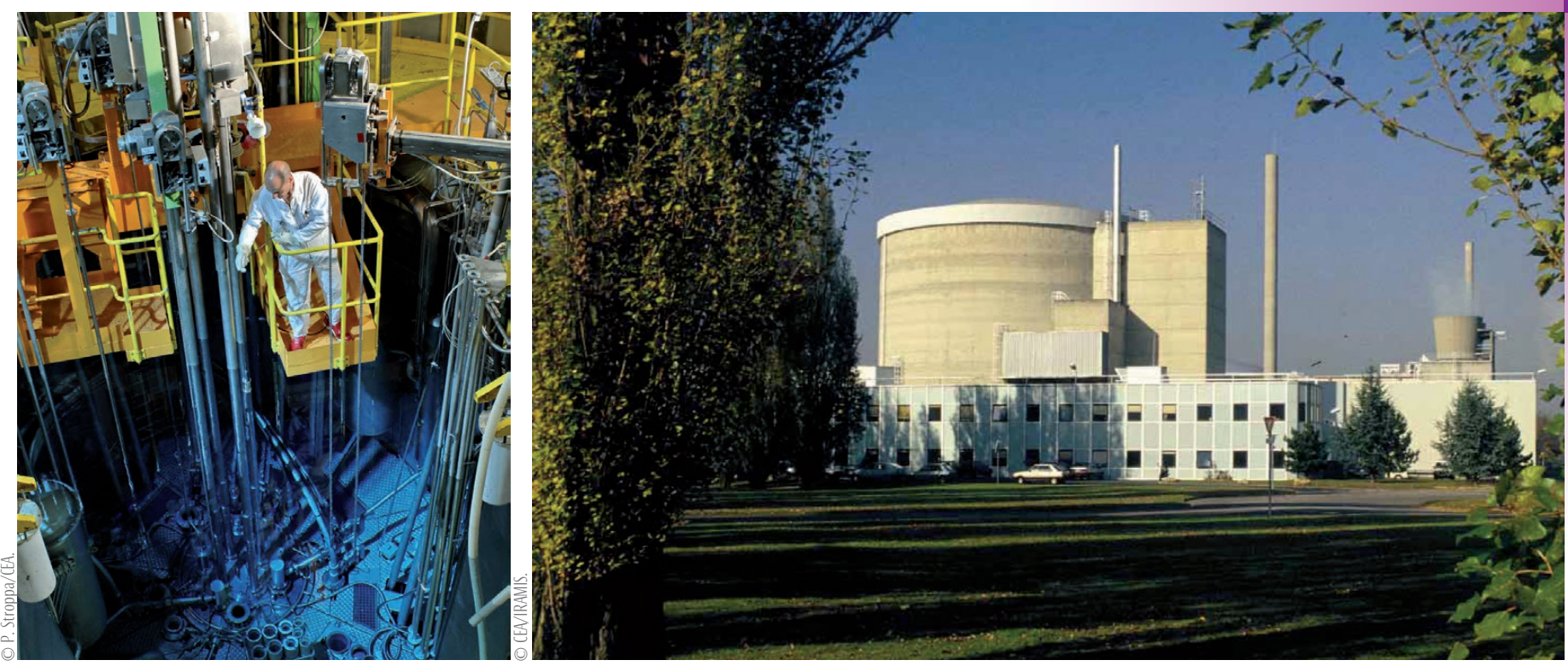

Le réacteur Orphée, source de neutrons du Laboratoire Léon Brillouin (LLB, UMR12 CEA-CNRS), au CEA/Saclay.

À gauche : Intervention au-dessus de la piscine du réacteur. À droite : le réacteur, vu du plateau de Saclay.

liberté pour prendre en compte toutes les informations apportées par les facteurs de structure. L'écart à l'isotropie, i.e. la dépendance angulaire de la contribution d'un pseudo-atome, est alors pris en charge par un développement sur un nombre limité de fonctions angulaires nommées " harmoniques sphériques réelles ", $y_{l, m \pm}(\theta, \varphi)$. À chaque atome correspond un repère local cartésien prédéfini en fonction de la symétrie atomique, sur lequel sont exprimés les harmoniques sphériques.

Par conséquent, la contribution globale d'un pseudo-atome $i$ à la distribution de densité dans le modèle de Hansen et Coppens s'écrit [1] :

$$
\begin{aligned}
& \rho_{i}^{\text {pseudo }}(\mathbf{r})=\rho_{i}^{\text {core }}(\mathbf{r})+P_{i}^{(\text {val. })} \kappa_{i}^{3} \rho_{i}^{(\text {val. })}\left(\kappa_{i} \mathbf{r}\right) \\
& +\sum_{l} \kappa_{i}^{\prime 3} R_{i, l}^{(\text {val. } .)}\left(\kappa_{i}^{\prime}{ }_{i} \mathbf{r}\right) \sum_{m=0}^{l} P_{i, l, m \pm} y_{l, m \pm}(\theta, \varphi)
\end{aligned}
$$

Le premier terme représente la contribution des électrons dits " de cœur", c'est-à-dire ceux qui sont les plus liés au noyau et qui, de ce fait, sont supposés n'intervenir que de manière négligeable dans la redistribution au moment de la création des liaisons chimiques. Le deuxième terme décrit les électrons de valence (dont le nombre effectif $P_{i}^{\text {(val.) }}$ conduit à une connaissance de la charge du pseudo-atome $i$ ) et intègre les effets moyens de contraction/expansion du nuage $\left(\kappa_{\mathrm{i}}\right)$. Le dernier terme rend compte des déformations angulaires, en particulier pour décrire l'ensemble des phénomènes de polarisation des régions atomiques sous l'effet des champs électriques créés par les atomes environnants. $P_{i, l, m \pm}$ est la population de l'harmonique sphérique $y_{l, m \pm}$, pour le pseudo-atome $i$.
Ce modèle possède ainsi un certain nombre de paramètres $\left\{\kappa_{i}, \kappa_{i}^{\prime}\right\},\left\{P_{i}^{(\text {val. })}\right.$, $\left.P_{i, l, m \pm}\right\}$, qui sont alors déterminés pour avoir le modèle de densité totale le plus fidèle possible à l'information fournie par l'ensemble des facteurs de structure mesurés [2].

\section{Les neutrons polarisés et le spin}

La diffraction d'un faisceau de neutrons par un monocristal résulte de deux interactions : celle des neutrons avec les noyaux atomiques, et l'interaction magnétique entre les moments magnétiques des neutrons et ceux des électrons non appariés dans le cristal. L'intensité diffractée pour chaque type d'interaction fait intervenir le facteur de structure relatif à l'arrangement périodique, respectivement celui des noyaux $\left(F_{\mathrm{N}}\right)$ caractérisés par une longueur de diffusion nucléaire $b_{\mathrm{N}}$ et celui des moments magnétiques électroniques $\left(F_{\mathrm{M}}\right)$ autour de chaque noyau (voir encadré 1 ).

Dans le cas d'un composé paramagnétique comme ceux que nous avons étudiés, les moments magnétiques électroniques sont alignés sous l'action d'un champ magnétique extérieur. Leur distribution dans la maille correspond à une grandeur vectorielle, la densité d'aimantation $\mathbf{m}(\mathbf{r})$, qui est liée à la densité d'électrons non appariés $s(\mathbf{r})$ par la relation $\mathbf{m}(\mathbf{r})=\mu s(\mathbf{r})$, où $\mu$ est un vecteur unitaire orienté selon la direction résultante des moments magnétiques dans le cristal. De même que les facteurs de structure électronique $F_{\mathrm{e}} \operatorname{DRX}_{(\mathrm{H})}$ sont les composantes de Fourier de la densité électronique $\rho(\mathbf{r})$, les facteurs de structure magnétique $F_{\mathrm{M}}{ }^{\mathrm{DNP}}(\mathrm{H})$ sont les composantes de la densité de $\operatorname{spin} s(\mathbf{r})$ :

$$
s(\mathbf{r})=\frac{1}{V} \sum_{\mathbf{H}}^{\infty} F_{M}^{D N P}(\mathbf{H}) \exp (-\mathbf{H} . \mathbf{r})
$$

Cette densité peut être positive ou négative selon la direction résultante des spins locaux $(\uparrow$ ou $\downarrow)$ par rapport au spin majoritaire $\uparrow$.

Si la diffraction des rayons $\mathrm{X}$ est maintenant bien établie comme mode privilégié de détermination de la densité de charge électronique dans les cristaux, en revanche la nécessité d'une meilleure compréhension des mécanismes responsables du magnétisme a encouragé le développement d'une approche similaire pour reconstruire la distribution d'aimantation au moyen de la diffraction de neutrons polarisés en $\operatorname{spin}$ (DNP) [3].

La diffraction de neutrons polarisés nécessite de placer l'échantillon paramagnétique dans un fort champ magnétique (jusqu'à 7 tesla au Laboratoire Léon Brillouin), de manière à orienter tous les moments magnétiques le long d'une direction privilégiée, choisie verticale (encadré 1). La mesure de diffraction est alors similaire à celle conduite avec des rayons $\mathrm{X}$, à une nuance près : pour chaque réflexion de Bragg, les intensités $I_{\uparrow}$ et $I_{\downarrow}$ correspondant aux neutrons incidents polarisés verticalement vers le haut $(\uparrow)$ ou vers le bas $(\downarrow)$ sont collectées séparément. C'est le rapport $R(\mathbf{H})=I_{\uparrow}(\mathbf{H}) / I_{\downarrow}(\mathbf{H})-$ nommé " rapport de flipping "- des deux signaux mesurés pour chaque vecteur $\mathbf{H}$ du réseau réciproque, qui est exploité et qui permet de déterminer avec une grande sensibilité les facteurs de structure magnétique $F_{\mathrm{M}}{ }^{\mathrm{DNP}}(\mathbf{H})$. La DNP est donc la méthode de choix pour déterminer les 


\section{Diffraction de neutrons polarisés}

encadré 1

L'une des principales difficultés de la détermination expérimentale des facteurs de structure magnétique $\left(F_{\mathrm{M}}^{\mathrm{DNP}}\right)$ pour un composé paramagnétique sous champ réside dans la séparation des signaux nucléaire et magnétique qui contribuent à l'intensité $I$ des mêmes raies de Bragg. Pour un faisceau de neutrons non polarisés, $l \propto\left(F_{N}{ }^{2}+\mathrm{F}_{M}{ }^{2}\right)$, sachant que $F_{M}<<F_{N}$ pour des composés faiblement magnétiques.

L'intérêt d'utiliser un faisceau de neutrons polarisés consiste à tirer profit, dans l'expression de l'intensité diffractée $I_{\uparrow, \downarrow} \propto\left|F_{\mathrm{N}} \pm F_{\mathrm{M}}\right|^{2}$, de l'existence d'un terme croisé $\pm 2 F_{\mathrm{N}} F_{\mathrm{M}}$ dont le signe varie en fonction de la direction de polarisation incidente $(\uparrow$ ou $\downarrow)$.

La grandeur expérimentale, qui est le rapport de flipping mesuré pour chaque raie de Bragg, contient alors un terme linéaire en $F_{\mathrm{M}} / F_{\mathrm{N}}$, d'où la sensibilité accrue de cette technique : $R(\mathbf{H})=\mathrm{I}_{\uparrow}(\mathbf{H}) / I_{\downarrow}(\mathbf{H})=\left[\left(F_{\mathrm{N}}+F_{\mathrm{M}}\right) /\left(F_{\mathrm{N}^{-}}-F_{\mathrm{M}}\right)\right]^{2} \cong 1+4 F_{\mathrm{M}} / F_{\mathrm{N}}$.

Le schéma du diffractomètre à neutrons polarisés $5 \mathrm{C} 1$ du Laboratoire Léon Brillouin, sur lequel ont été effectuées les expériences décrites dans cet article, est montré sur la figure E1.

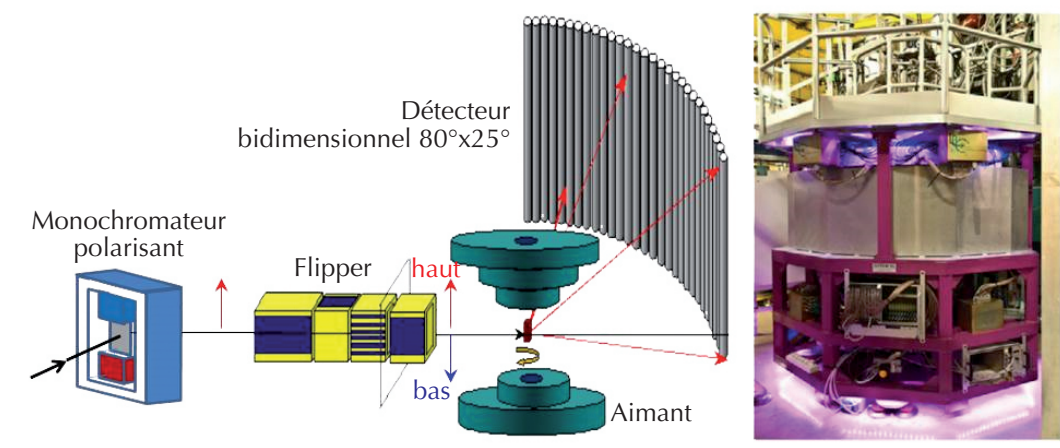

E1. La polarisation des moments magnétiques des neutrons est réalisée par diffraction du faisceau par un monochromateur polarisant (cristal ferromagnétique de CuMnAl), qui ne diffracte qu’un seul état de spin pour la raie (111), pour laquelle on a $F_{M}=-F_{N}$. La direction de polarisation du faisceau incident de neutrons selon la verticale est alternée grâce à un flipper, dispositif constitué d'un électro-aimant et d'une plaque supraconductrice. L'échantillon monocristallin est placé dans un aimant supraconducteur permettant d’appliquer un champ magnétique vertical de 7 teslas, à une température minimale de 1,5 K. Les intensités diffractées pour les deux états initiaux $(\uparrow)$ et $(\downarrow)$, dans la direction de polarisation du faisceau de neutrons incident sont collectées grâce à un détecteur bidimensionnel d'ouverture $25^{\circ} \times 80^{\circ}$

\section{\>}

régions de la maille cristalline où une différence de spin électronique existe et comment elle est distribuée.

Comme, hélas, il y a de moins en moins de sources de neutrons et que la collecte des intensités diffractées est plus longue que son équivalent avec des rayons $\mathrm{X}$, les contraintes d'une collection de données par DNP sont plus fortes que par DRX. Cependant, à la différence de cette dernière qui met en jeu tous les électrons, qu'ils soient de cœur ou de valence, les facteurs de structure magnétiques ne contiennent que la contribution d'électrons non appariés situés sur des couches de valence. Il n'est alors pas nécessaire d'explorer un aussi large domaine de vecteurs $H$. Ainsi, la DNP est généralement limitée au domaine des vecteurs du réseau réciproque pour lesquels une contribution magnétique significative est attendue, soit $H_{\max } \leq 0,5 \AA^{-1}$ pour les métaux de transition porteurs d'électrons non appariés sur la couche $3 d$.

proque exploré est limité en raison d l'encombrement autour de l'échantillon, lié à la présence d'un électro-aimant. Une contrainte additionnelle est que la précision expérimentale des rapports de flipping est conditionnée à l'existence d'une forte intensité de la réflexion de Bragg provenant de la contribution (non magnétique) des noyaux. Il en résulte que tout l'espace réciproque ne peut alors être exploré. Ainsi, dans l'exemple reporté dans cet article, environ 20\% seulement des réflexions dans le domaine $H_{\max } \leq 0,5 \AA^{-1}$ sont mesurées par DNP alors que plus de $98 \%$ des raies sont collectées par DRX dans ce même domaine.

En dépit de ces difficultés, la DNP est une méthode unique pour visualiser la densité de spin (voir section suivante) et comprendre les mécanismes d'interaction magnétique dans les cristaux [4].

\section{De la nécessité de marier les techniques pour atteindre une distribution électronique résolue en spin}

De ce qui précède, il apparait clairement que les méthodes DRX et DNP sont complémentaires : la diffraction des rayons $\mathrm{X}$ permet une reconstruction de la distribution de tous les électrons - la densité de charge $-\rho(\mathbf{r})$, tandis que la DNP contient des informations qui donnent accès à la distribution des électrons non appariés responsables du magnétisme - la densité de spin - $\mathrm{s}(\mathbf{r})$. Ces deux quantités peuvent être exprimées en termes de densités d'électrons résolues en spin, $\rho_{\uparrow}(\mathbf{r})$ et $\rho_{\downarrow}(\mathbf{r})$, et inversement : $2 \rho_{\uparrow}(\mathbf{r})=\rho(\mathbf{r})+s(\mathbf{r})$, et $2 \rho_{\downarrow}(\mathbf{r})=\rho(\mathbf{r})-s(\mathbf{r})$. L'analyse combinée des expériences de diffraction de rayons $\mathrm{X}$ à haute résolution et de neutrons polarisés donne donc un accès unique à des densités électroniques résolues en spin pour des cristaux ayant des propriétés magnétiques intéressantes. Cependant, aucune analyse combinée réussie n'a été rapportée jusqu'à ce jour. On devine qu'une raison essentielle réside dans la conjonction de deux obstacles majeurs : l'absence d'un modèle commun du comportement des électrons et la difficulté de trouver une juste pondération entre les jeux de données issus des différentes expériences.

\section{Le modèle spin-split et son affinement joint}

L'exploitation simultanée des données issues de la diffraction des rayons $\mathrm{X}$ à haute résolution et de celle des neutrons polarisés, a conduit à modifier le modèle de Hansen et Coppens pour obtenir une forme adaptée à une description résolue en spin. Dans ce nouveau modèle, les sites atomiques pour lesquels on est fondé à attribuer un comportement magnétique, sont décrits - dans leur composante de valence - par un double pseudo-atome, chacun décrivant les électrons associés à un état de spin particulier (modèle spin-split).

Dans la mesure où, finalement, dans une molécule un nombre restreint de sites atomiques possèdent une densité significative d'électrons non appariés, le nombre de paramètres du modèle ne se trouve que modérément augmenté par rapport au modèle habituel de densité de charge. 
Pour un site atomique porteur d'un moment magnétique, la contribution du double pseudo-atome associé s'écrit alors :

$$
\begin{aligned}
& \rho_{i}^{\text {core }}(\mathbf{r})+P_{i}^{\uparrow \vee v a l} \kappa_{i}^{\uparrow 3} \rho_{i}^{v a l}\left(\kappa_{i}^{\uparrow} \mathbf{r}\right)+P_{i}^{\downarrow v a l} \kappa_{i}^{\downarrow 3} \rho_{i}^{\text {val }}\left(\kappa_{i}^{\downarrow} \mathbf{r}\right) \\
& +\sum_{l} \kappa_{i}^{\prime \uparrow 3} R_{i, l}^{\uparrow v a l}\left(\kappa_{i}^{\uparrow \uparrow} \mathbf{r}\right) \sum_{m=0}^{l} P_{i, l, m \pm}^{\uparrow} y_{l, m \pm}(\theta, \varphi) \\
& +\sum_{l} \kappa_{i}^{\prime \downarrow 3} R_{i, l}^{\downarrow v a l}\left(\kappa_{i}^{\downarrow \downarrow} \mathbf{r}\right) \sum_{m=0}^{l} P_{i, l, m \pm}^{\downarrow} y_{l, m \pm}(\theta, \varphi)
\end{aligned}
$$

où $P_{\uparrow}$ et $P_{\downarrow}$ traduisent respectivement les populations électroniques de spin majoritaire $\uparrow$ et de spin minoritaire $\downarrow$ présentes sur le site considéré.

Ainsi, le défi consiste à déterminer, pour chaque site atomique, les $P_{\uparrow}$ et $P_{\downarrow}$, ainsi que les coefficients de contraction/ dilatation radiale $\kappa_{\uparrow}$ et $\kappa_{\downarrow}$, en exploitant au mieux, et simultanément, les différents jeux de données de diffraction, jouant ainsi sur la complémentarité des méthodes et le renforcement mutuel des informations expérimentales.

Trouver un moyen équilibré de rassembler des données expérimentales de différentes origines et de les affiner ensemble par moindres carrés représente une véritable difficulté, et le schéma de pondération utilisé doit être adéquat $[5,6]$.

\section{Une première mise en pratique sur un aimant moléculaire}

Pour valider notre approche, il fallait utiliser un cristal possédant peu de centres magnétiques et dont les données de diffraction ne posaient pas de problèmes particuliers.

Nous avons choisi un complexe dinucléaire de cuivre (II) $(s=1 / 2)$ où les atomes de cuivre sont couplés de manière ferromagnétique par des ponts $\mathrm{N}_{3}{ }^{-}$, appelés azido: $\mathrm{Cu}_{2} L_{2}\left(\mathrm{~N}_{3}\right)_{2}$ (fig. 1 );

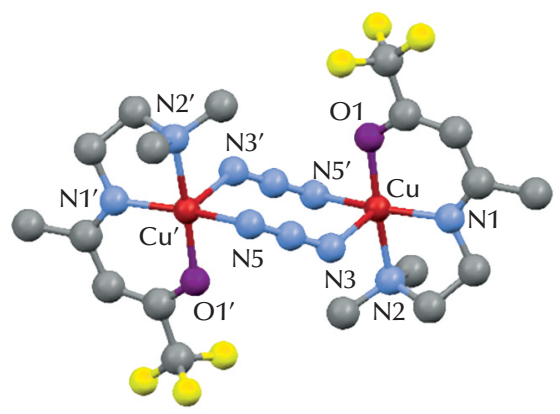

1. Complexe de cuivre di-azido. Vue de la molécule $\mathrm{Cu}_{2} \mathrm{~L}_{2}\left(\mathrm{~N}_{3}\right)_{2}$ : les atomes d'azote sont représentés en bleu, ceux d'oxygène en violet, ceux de carbone en gris et ceux de fluor en jaune. Les atomes de cuivre sont en rouge. Les atomes d'hydrogène ne sont pas représentés, pour des raisons de clarté.
$L$ est un ligand. Le mécanisme responsable de l'interaction magnétique $\mathrm{Cu} . . . \mathrm{Cu}$ a longtemps été l'objet d'interrogations. Il a finalement été accepté, en partie grâce à la diffraction de neutrons polarisés, que le couplage ferromagnétique de tels complexes est dû à la quasi-orthogonalité des orbitales magnétiques centrées sur les atomes de cuivre, alors qu'un recouvrement important entre ces mêmes orbitales donnerait un couplage antiferromagnétique. Ce composé est bien adapté à notre test de validité, car sa structure cristalline est formée de complexes centrosymétriques neutres dinucléaires de cuivre pentacoordonnés (distances $\mathrm{Cu}-\mathrm{Cu}=5,068 \AA$ ), bien isolés les uns des autres. Les groupes azido relient les ions $\mathrm{Cu}$ dans un mode asymétrique, de sorte que les distances $\mathrm{Cu}-\mathrm{N} 3=2,000 \AA$ et $\mathrm{Cu}-\mathrm{N} 5=2,346 \AA$ sont clairement différentes [7].

\section{Première reconstruction de la densité électronique résolue en spin, et comparaison avec des calculs quantiques ab initio}

Le modèle spin-split, affiné à partir des données de DRX collectées sur le cristal moléculaire à une température de $10 \mathrm{~K}$ et des données de DNP obtenues à $2 \mathrm{~K}$ sous un champ magnétique appliqué de $6 \mathrm{~T}$, a permis de construire les cartes expérimentales de densité de charge et de densité de spin présentées dans la figure 2. La figure 2a représente la densité électronique statique de déformation consécutive à la formation des liaisons moléculaires. Elle est obtenue en soustrayant une densité d'électrons modèle bâtie à partir d'atomes indépendants, neutres et sphériques, à la distribution expérimentale d'électrons calculée à partir du modèle de Hansen et Coppens $\left(\rho(\mathbf{r})=\rho_{\uparrow}(\mathbf{r})+\rho_{\downarrow}(\mathbf{r})\right)$. La figure $2 \mathrm{~b}$ montre la distribution de spin expérimentale $\left(s(\mathbf{r})=\rho_{\uparrow}(\mathbf{r})-\rho_{\downarrow}(\mathbf{r})\right)$.

La carte de densité de déformation de charge établie dans le plan défini par les atomes N1, Cu1 et N2 (fig. 2a), issue des informations conjointes de DRX et DNP, montre une redistribution des électrons en accord avec la théorie du champ cristallin (encadré 2). Le repère local du cuivre a été choisi tel que $\mathrm{x}$ soit dirigé vers $\mathrm{N} 1$, y perpendiculaire à la direction $\mathrm{Cu}-\mathrm{N} 1$ (donc vers $\mathrm{N} 2$ ), et $\mathrm{z}$ dans la direction N3. On notera la réorganisation des électrons localement décrits par des

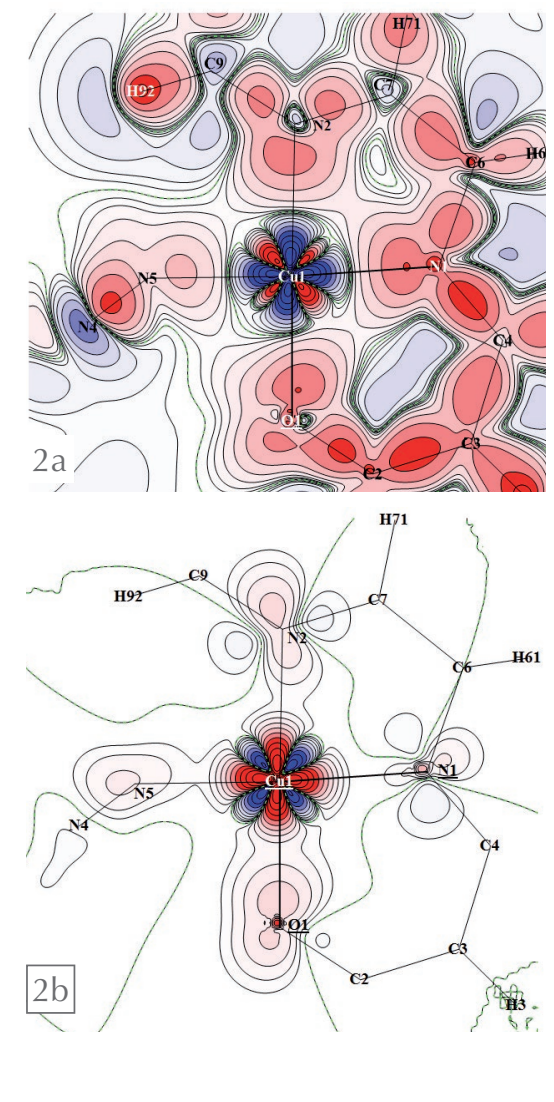

2. Cartes expérimentales des densités de charge et de spin obtenues à l'aide de la stratégie d'affinement joint au modèle spin-split.

(a) Carte de déformation statique de la densité de charge. Les contours d'isodensité sont tracés pour $\pm 0,01 \times 2^{n}$ électron $/ \AA^{3}$, avec $n=0$ à 13 . En rouge, les valeurs positives, celles qui correspondent à un excès d'électrons. En bleu, les valeurs négatives correspondant à une déplétion d'électrons.

(b) Carte de densité de spin. Les lignes d'isodensité sont tracées pour $\pm 0,01 \times 2^{n} \mu_{B} / \AA^{3}$, avec $n=0$ à 13 , le spin majoritaire $(\uparrow)$ en rouge, et le spin minoritaire $(\downarrow)$ en bleu.

orbitales de type $d$ autour des métaux dépeuplement de l'orbitale $d_{x^{2}-\gamma^{2}}$ qui pointe vers les ligands, et peuplement de l'orbitale $d_{x y}$ qui pointe dans les directions bissectrices - ainsi que les paires «libres » d'électrons des atomes $\mathrm{N}$ et $\mathrm{O}$ des ligands, qui interagissent avec les atomes de cuivre via des liaisons de coordination. La densité de spin, là aussi bâtie à partir des informations $\mathrm{X}$ et $\mathrm{N}$ (fig. 2b), montre une distribution d'électrons de type $d_{x^{2}-y^{2}}$ autour du cuivre, avec des maxima de spin majoritaire $\uparrow$ dirigés vers les atomes $\mathrm{N}$ et $\mathrm{O}$, et des maxima de spin minoritaire $\downarrow$ dans la direction bissectrice. Une telle population est en parfait accord avec le dépeuplement partiel de l'orbitale $d_{x^{2}-\gamma^{2}}$ observé sur la densité électronique de déformation. On retrouve très peu de densité de spin 

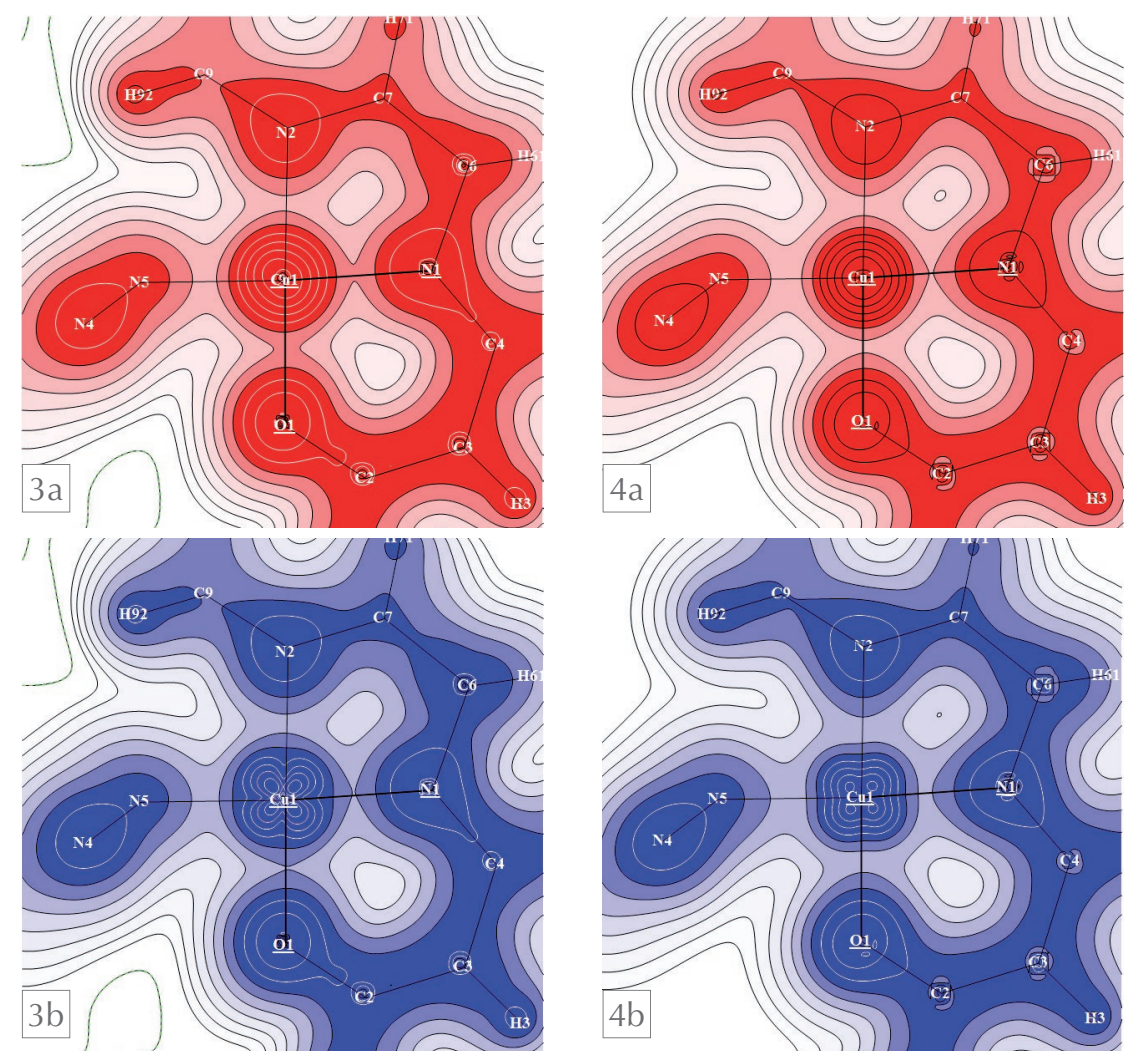

3. Densités de probabilité de présence expérimentales des électrons de valence résolues en spin par la méthode d'affinement joint du modèle spin-split. Les cartes sont tracées dans le plan formé par les noyaux Cu1-N1-01, avec des contours d'isodensité tous les $0,01 \times 2^{n}$ électron $/ A^{3}(n=0$ à 12) La densité d'électrons de spin majoritaire ( $\uparrow$ ) est représentée en rouge (figure a), et celle de spin minoritaire $(\downarrow)$ en bleu (figure b).

4. Mêmes observables physiques que dans la figure 3, obtenues par calculs quantiques ab initio.

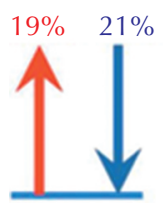

$z^{2}$

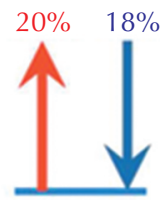

$X Z$

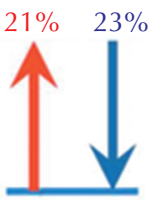

$y z$

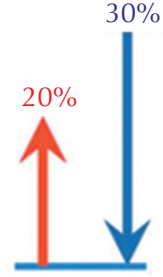

xy
5. Représentation schématique de populations d'orbitales $d$ sur les sites des atomes de cuivre en fonction des natures de spin. Les tailles des flèches sont proportionnelles aux populations de spins respectives. Les spins majoritaires ( $\uparrow$, en rouge) sont répartis de manière isotrope sur les cinq orbitales, tandis que l'anisotropie des spins minoritaires ( $\downarrow$, en bleu) est marquée pour les orbitales $d_{x y}$ et $d_{x^{2}-y^{2}}$.

\begin{tabular}{|c|c|c|}
\hline & Expérience & Théorie \\
\hline$q \uparrow$ (électrons) & 14,56 & 14,29 \\
\hline$q \downarrow$ (électrons) & 13,78 & 13,66 \\
\hline$\Delta q$ (électrons) & 0,78 & 0,63 \\
\hline$\mu_{\uparrow}$ (Debye) & 0,063 & 0,102 \\
\hline$\mu_{\downarrow}$ (Debye) & 0,072 & 0,130 \\
\hline
\end{tabular}

Tableau 1. Valeurs déduites des densités expérimentales et théoriques pour les populations électroniques de spin $\uparrow(\mathbf{q} \uparrow)$ et $\downarrow(\mathbf{q} \downarrow)$, y compris les électrons de cœur, et pour les moments dipolaires électroniques ( $\mu$, en Debye) correspondants.

\section{\>}

dans le voisinage des atomes de ligand. Pour une description plus détaillée de la procédure d'affinement, on pourra se référer à l'article de Deutsch et al. [8].

Une première distribution de densité d'électrons résolue en spin, obtenue à partir de cette analyse conjointe, est représentée sur la figure 3. Il est clair que le modèle de spin-split permet de discriminer les distributions des électrons de spins majoritaire et minoritaire. À titre de comparaison (fig. 4), les calculs quantiques ab initio basés sur la théorie de la fonctionnelle de la densité (DFT) ont été réalisés sur une molécule isolée dans sa géométrie expérimentale. Les distributions théoriques et expérimentales sont extrêmement similaires, au point que les différences sont à peine visibles au niveau du contour dessiné.

La reconstruction expérimentale arrive parfaitement à mettre en évidence la sphéricité de distribution de spin majoritaire (en rouge) dans le voisinage du noyau de cuivre, ainsi que l'anisotropie de la distribution de spin minoritaire (en bleu) pour pointer le long de la bissectrice des ligands. Il apparaît de manière très nette que l'essentiel de cette anisotropie électronique autour de l'atome de cuivre doit être attribuée aux électrons de spin minoritaire $(\downarrow)$. Ceci est d'ailleurs confirmé par une analyse de la population des orbitales $d$ (encadré 2 ) des sites de cuivre. Cette estimation est schématisée sur la figure 5 . On constate que 30\% des électrons de spin minoritaire se trouvent décrits localement par une fonction d'onde de type $d_{\mathrm{xy}}$, alors que la fonction d'onde $d_{\mathrm{x}^{2}-\mathrm{y}^{2}}$ n'en contient qu'environ $9 \%$, les fonctions restantes $d_{\mathrm{xz}}, d_{\mathrm{yz}}$ et $d_{\mathrm{z}^{2}}$ étant toutes presque identiquement peuplées. L'orbitale $d_{\mathrm{x}^{2}-\mathrm{y}^{2}}$, la plus déstabilisée (puisqu'en face des ligands), est donc peuplée d'électrons majoritaires et minoritaires ; ceci est en accord avec une covalence partielle de la liaison métal-ligand.

Une conséquence importante et originale de ce modèle résolu en spin est que le nuage électronique de spin majoritaire $(\uparrow)$ est plus contracté (d'environ 5\%) autour du noyau de cuivre que celui du spin minoritaire $(\downarrow)$. Ceci est traduit dans le modèle spin-split par une différence des coefficients de contraction-dilatation $\left(\boldsymbol{\kappa}^{\uparrow}=0,998, \boldsymbol{\kappa}^{\downarrow}=0,943\right)$, en accord avec des prédictions théoriques faites dans les années 1960 puis 1980 [9, 10]. 
La répartition de la densité électronique des métaux de transition peut être prédite par la théorie du champ cristallin. Rappelons que la structure électronique de valence des éléments de transition dans leur état fondamental se décrit à partir d'électrons $4 s$ de symétrie sphérique, et d'électrons $3 d$. Comme le montre la figure E2, ces derniers peuvent se répartir dans cinq orbitales de même énergie : $d_{z^{2}}, d_{x z^{\prime}} d_{y z}, d_{x^{2}-y^{2}}, d_{x y}$.

Chaque orbitale $d$ est très anisotrope et chaque lobe pointe dans des directions d'espace différentes; la densité électronique associée, calculée par le produit de la fonction d'onde de l'orbitale par sa partie conjuguée, est bien sûr positive ou nulle partout, mais conserve les mêmes orientations et symétries. Chaque orbitale $d$ peut être peuplée par 0, 1 ou 2 électrons. Lorsqu'un composé de coordination se forme avec un métal de transition et des ligands polaires ou anioniques, il y a répulsion électrostatique des électrons $d$ situés en face de ces ligands; les orbitales $d$ ne sont plus équivalentes du point de vue énergétique : on dit qu'il se produit une levée de dégénérescence. On observera alors une population préférentielle des orbitales $d$ qui ne pointent pas vers les ligands et qui sont celles de plus basse énergie.

Pour le complexe de Cu II étudié, il y a 9 électrons $3 d$ et la coordination est pentagonale déformée.

On a donc trois niveaux de dégénérescence. La théorie du champ cristallin nous dit que l'orbitale $d_{x^{2}-y^{2}}$, qui se trouve en face de ligands de charge négative, est déstabilisée et donc dépeuplée. Mais l'affinement joint va plus loin, car il montre que les orbitales $d_{z^{2}}$ $d_{\mathrm{xz}}$ et $d_{\mathrm{yz}}$ sont peuplées de manière identique, donc d'énergie quasi équivalente, et ne participent pas aux propriétés magnétiques ; l'orbitale $d_{x y}$ récupère la densité électronique perdue par $d_{x^{2}-y^{2}}$ et est l'orbitale la plus stable. En conséquence, ces deux dernières orbitales sont magnétiques.

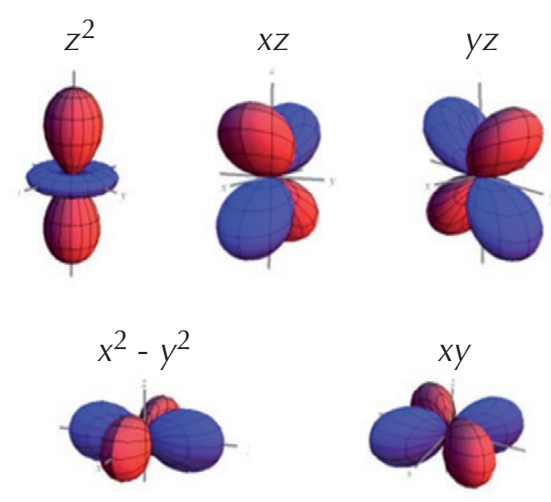

E2. Représentation schématique des symétries et anisotropies des cinq orbitales $3 d$ des éléments de transition (bleu : fonction d'onde négative, rouge : fonction d'onde positive).
Une analyse topologique de la densité électronique résolue en spin a également été réalisée suivant l'approche "Atoms in Molécules" [11]. Un bon accord entre la théorie et l'expérience est observé. Par exemple, les charges nettes obtenues en intégrant les distributions des électrons de spin majoritaires et minoritaires sur les bassins atomiques du cuivre (i.e. volume effectif de l'atome en interaction avec ses voisins) (tableau 1) montrent que si, expérimentalement, on observe un excès de 0,78 électron de spin majoritaire ( $\uparrow$ ), cette différence est par DFT de 0,63. Les mêmes tendances (mais avec un décalage en valeur absolue plus net) se retrouvent sur les moments dipolaires attribués aux sites métalliques.

\section{Conclusion et perspectives}

Il ressort de cette étude que la stratégie d'affinement joint à partir de données issues des diffractions des rayons $\mathrm{X}$ à haute résolution, des neutrons et des neutrons polarisés, permet, grâce au modèle spin-split, de reproduire précisément les caractéristiques de la distribution de probabilité de présence des électrons en tenant compte de leur état de spin. On montre, dans l'exemple choisi, qu'il est alors possible de distinguer les différences de comportement des électrons de spin majoritaire et minoritaire dans leur redistribution spatiale autour des noyaux de cuivre, consécutive à la formation des liaisons chimiques.

Pour la première fois, le modèle de la densité électronique résolu en spin apporte la confirmation expérimentale d'une contraction/expansion des distributions radiales des électrons périphériques dépendant des états de spin. La très grande similitude, même au niveau de l'analyse topologique, entre cette reconstruction expérimentale et celle obtenue suivant une méthode de calcul quantique $a b$ initio, conforte ces deux approches. Un tel accord permet d'explorer théoriquement par DFT des molécules et complexes qui ne peuvent être facilement cristallisés ou étudiés expérimentalement.

En définitive, cette première étude montre qu'il est maintenant possible d'accéder expérimentalement aux densités d'électrons selon leurs états de spin, ce que les méthodes standard monotechniques qui prévalaient jusque-là ne permettaient pas. La méthode d'affinement multiple peut être appliquée à n'importe quel matériau cristallin magnétique, minéral ou organique. Dans une perspective plus large, cette méthode ouvre la voie à la combinaison de différentes expériences de diffusion, qui mèneront à une description plus précise et fiable du comportement électronique dans les solides cristallins (diffraction magnétique des rayons $\mathrm{X}$, diffusion Compton magnétique...).

\section{Références}

1 N.K. Hansen et P. Coppens, "Testing aspherical atom refinements on small-molecule data sets" Acta Cryst. A 34 (1978) 909-921.

2- C. Gatti et P. Macchi (eds.), Modern Charge Density Analysis, Springer (2012).

3• P.J. Brown et al., "Spin densities in free radicals" J. Magn. Magn. Mater. 14 (1979) 289.

4• Y. Pontillon et al., "Experimental Spin Density in a Purely Organic Free Radical: Visualisation of the Ferromagnetic Exchange Pathway in p-(Methylthio) phenyl Nitronyl Nitroxide, Nit(SMe)Ph" Chem. Eur. J., 5 (1999) 3616-3624.

5• B. Bell et al., "A relative weighting method for estimating parameters and variances in multiple data sets" Comp. Stat. and Data Anal., 22 (1996) 119-135.

6• J.-M. Gillet et al., "Joint refinement of a local wave-function model from Compton and Bragg scattering data" Phys. Rev. B 63 (2001) 235115.

7• C. Aronica et al., Chem. Eur. J., 13 (2007) 36663674.

$8 \bullet$ M. Deutsch et al., "First spin resolved electron distributions in crystals from combined polarized neutron and X-ray diffraction experiments" IUCr.., 1 (2014) 194-199.

9• R.E. Watson et A.J. Freeman, “Crystalline Field and Spin Polarization Effects on Electron Densities and Magnetic Form Factors" Phys. Rev. 120 (1960) 1134-1141.

10• P.J. Becker et P. Coppens, "About the simultaneous interpretation of charge and spin density data" Acta Cryst. A 41 (1985) 177-182.

11• R.W.F. Bader, Atoms in Molecules. A Quantum Theory, Oxford University Press (1990). 